Case Reports

\title{
Alice in wonderland syndrome; a migraine variant
}

\author{
Jithangi Wanigasinghe ${ }^{1}$ \\ Sri Lanka Journal of Child Health, 2012; 41(1): 40-41
}

(Key words: Alice in wonderland syndrome; migraine variant)

\begin{abstract}
Alice in wonderland syndrome (AWLS) is the episodic occurrence of a collection of visual illusions that are similar to those described in the famous Alice stories written by Louise Carol. The patient experiences visual phenomena as the predominant symptom but rare auditory, sensory and motor illusions are reported. It is known to follow several viral infections; however, headache during events, past history of migraine, family history of migraine in these patients, confirm migraine (migraine variant) as an underlying aetiology.
\end{abstract}

\section{Case history}

A 12 year old girl presented with a one month history of episodes of unusual phenomena which occurred when concentrating or trying to fall sleep. She had visual illusions occurring in the form of altered shape and size. The objects around her appeared too small (micropsia) or too large and closer (peliopsia) and this often made her feel frightened. She sometimes saw her own hand several times larger or extremely small in size. During these episodes she might experience various other phenomena. The most distressing of these was a feeling of her actions being ñspeeded upò The others included intermittent tinnitus in both ears. Each of these episodes might last as long as 20 minutes, and might be experienced several times a day. In the past she has suffered from infrequent episodes of migrainous headache, but with the onset of the current symptoms the frequency of headache has increased but not necessarily only during the unusual ñeventsò. The onset of these symptoms did not follow a viral fever or other recent illness. She gave no history of epilepsy. Neurological and eye examinations were completely normal. She was negative for EpsteinBarr viral antibodies. Her electroencephalogram during the resting state was normal. She did not undergo neuro-imaging.

${ }^{1}$ Senior Lecturer in Paediatrics \& Honorary
Consultant Paediatric Neurologist, Faculty of
Medicine, University of Colombo

(Received on 25 March 2011: Accepted on 29 April 2011)
As there was no spontaneous improvement in the frequency of ñeventsò over the next 4 weeks, she was commenced on anticonvulsant therapy (carbamazepine). She reported minimal improvement and therefore was commenced on anti-migraine prophylactic therapy. After a four week interval she reported marked reduction in the frequency of events.

\section{Discussion}

Migraine variants (MVs) may occur in 3-5\% of those suffering from migraine and usually affect children and young adults ${ }^{1}$. AWLS is a rare form of $\mathrm{MV}^{2}$. The other neurological manifestations described in AWLS (apart from those experienced by this patient) include shrinking in size to that of index finger (Lilliputian syndrome), movement of objects up and down, inability to recognise colour (acromatopsia), persisting or recurrence of visual images even after removing the stimulus (palinopsia), inability to recognise faces (prosopagnosia) etc. In this syndrome the complex perceptual changes are predominantly visual but auditory phenomena with voices heard faint and distant or close and louder and tinnitus are reported. These perceptual changes are not hallucinatory as the affected knows that distorted objects or body images are not real.

The pathophysiological mechanism associated with migraine variants is called a cortical spreading depression (CSD). This is a slow cortical depolarisation that spreads from one area of the cortex to that which is contiguous. This results in an initial oligaemia/ischaemia followed by hyperaemia. Such perfusion abnormalities during episodes of AWLS have been shown using Single Positron Emitting Tomography (SPECT) studies ${ }^{3}$.

The outcome of AWLS associated with viral infections is generally considered transient. However, resolution over weeks to months is reported. Rare cases of long term recurrences are reported. In those where it occurs due to a MV, excellent response has been shown with GABAergic effects of sodium valproate or with other anti-migraine treatments but need for long term therapy is indicated ${ }^{4}$. 


\section{References}

1. Lipton RB, Bigal ME. Migraine: epidemiology, impact and risk factors for progression. Headache 2005; 45(Suppl 1):S3-S13

http://dx.doi.org/10.1111/j.15264610.2005.450 $\underline{1001 . \mathrm{x}}$

2. Rolak LA. Literary neurologic syndrome: Alice in Wonderland. Archives of Neurology 1991; 48(6):649-51.

http://dx.doi.org/10.1001/archneur.1991.00530 180107025
3. Gencoglu EA et al. Brain SPECT Findings in a patient with Alice in Wonderland syndrome. Clinical Nuclear Medicine 2005; 30: $758 і ̈ 9$.

http://dx.doi.org/10.1097/01.rlu.0000182278.1 $\underline{3389 . \mathrm{a} 3}$

4. Hamed S A. A migraine variant with abdominal colic and Alice in wonderland syndrome: a case report and review. BMC Neurology 2010; 10(2). Available from:

http://www.biomedcentral.com/1471-2377/10/2 